\title{
Performance comparison of Stability Enhanced AODV and Stability Enhanced AOMDV Protocol for MANETs
}

\author{
Chintan Bhavsar \\ Assistant Professor, G.H Patel College of Engineering \& Technology, V. V Nagar, Gujarat, India \\ Sonal Belani \\ Trainee Lecturer, MBICT, V. V Nagar, Gujarat, India
}

\begin{abstract}
A Mobile Ad hoc network is set nodes, which communicates without any infrastructure. The topology for such networks is dynamic in nature. The nodes in MANETs have limited and shared bandwidth and limited battery power are. Such limitations make design of a mobile ad hoc network a very challenging task. To design a routing protocol for such dynamic topologies is a challenging task. There is a high possibility of a route failure due to constant movement of nodes. When a route fails, route discovery procedure has to be started to find a new route. Increase in number of route discoveries increases the routing overhead and delay. AODV protocol finds route which contains minimum hop, without considering stability of the link. The existing AOMDV protocol has provision of multiple routes. The route in existing AOMDV is found on minimum hop basis without considering strength of a link. We can find more stable routes, if we use received signal strength as a metric to find a route. Provision of stable route may increase performance of the network. In this paper we compare performance of stability enhanced AOMDV and stability enhanced AODV, both considers received signal strength as a decisive parameter to find a route.
\end{abstract}

Keywords- MANET, Routing, Stability, Cross-Layer, Received signal strength, AODV, AOMDV

\section{INTRODUCTION}

A mobile ad hoc network (MANET) is a set of mobile devices connected by wireless links. It is a network which is infrastructure less and dynamic in nature. Each device in MANETs frequently changes its links to other devices as it is free to move any direction. Each node must be equipped to route the traffic. To efficiently route the traffic each device should continuously maintain the routing related information. The mobile nodes of MANETs have limited transmission power which also affects topology of the network [1].

One of the major problems in ad-hoc networking is routing of data packets because, the topology is not predetermined and it does not have centralized control. Hence, routing in ad-hoc networks can be viewed as a challenge due to the frequently changing topology.

There are set of on-demand or reactive routing protocols in MANETs which creates the routes as and when required. Route discovery mechanism is started when there is a requirement to find a route. They only maintain the routes that are currently in use to minimize control overhead and routing load [1].

Existing routing protocols in ad-hoc networks utilize the single route that is built for source and destination node pair. Due to node mobility, node failures and the dynamic characteristics of the radio channel, links in a route may become temporarily unavailable, making the route invalid [1].

The overhead of finding alternative routes mounts along with additional packet delivery delay. This problem can be solved by use of multiple paths between source and destination node pairs, where one route can be used as the primary route and the rest as backup. Performance can be adversely affected by high route discovery latency and frequent route discovery in dynamic networks. This can be reduced by computing multiple paths in a single route discovery attempt. Multiple paths can be formed for both traffic sources and intermediate nodes with new routes being discovered only when needed, reducing route discovery latency and routing overheads. Multiple paths can also balance network load by forwarding data packets on multiple paths at the same time.

The other way of reducing the routing overhead is to find a route which remains active for longer time. A more stable path could be found that would reduce the number of route failures. A stable route would lead to less number of route discoveries and in turn reduce the routing overhead [15].

We can also find multiple paths such that they are stable. There can be combination of multiple paths with added stability to those paths. 
In this paper, we compare two approaches.

(1) AODV with Enhanced Stability

(2) AOMDV with Enhanced Stability.

\section{BACKGROUND}

On- demand route discovery is the main principle of reactive routing protocols. On- demand route discovery means creating routes as and when required. They generally find fresh routes, rather than using stored routes. Our discussion is limited to two on-demand ad-hoc routing protocols, AODV and AOMDV, as follows.

\section{a. Ad-hoc On-Demand Distance Vector Routing (AODV)}

AODV is a reactive protocol that discovers routes as and when required. It uses an on-demand route discovery mechanism. It maintains one entry per destination in traditional routing tables. RREQ (route request) packets are broadcasted by sender whenever there is a need to find a route. The RREQ is received by neighbouring nodes, which forwards it to their neighbours. RREQ packet travels through intermediate node to its intended recipient. Destination node sends RREP (route reply) packet upon receiving RREQ packet. To prevent routing loops and to maintain the freshness of route AODV maintains sequence numbers for each destination [11]. All routing packets carry these sequence numbers. AODV maintains timer based states in each node to remove the unused or older entries in routing table. List of precursors indicates set of neighbouring nodes. Such precursor lists are maintained for each routing table entry. Whenever the next-hop links breaks the nodes in a precursor list are notified by RERR (route error) packet .This Packet gets forwarded by each predecessor node to its Predecessors, effectively erasing all routes using the broken link [11]. The advantages of AODV are that less memory space is required as information of only active routes are maintained, in turn increasing the performance, while the disadvantage is that this protocol is not scalable and in large networks it does not perform well and does not support asymmetric links.

\section{b. Ad-hoc On-demand Multipath Distance Vector Routing (AOMDV)}

Ad-hoc On-demand Multipath Distance Vector Routing (AOMDV) [12] protocol is an extension to the AODV protocol with provision of multiple paths. It finds multiple paths such that they are loop-free and link disjoint paths. There can be multiple next hops for the same destination with same sequence number, which helps in keeping track of a route. AOMDV maintains an advertized hop count for each destination at node. Advertized hop count is nothing but maximum hop count for particular destination. Each duplicate route advertisement received by a node defines an alternate path to the destination. Advertized hop counts ensures Loop freedom for multiple routes. Alternative paths are only considered if they have less hop count than advertized hop count. For same sequence number advertised hop count does not change [12]. The next-hop list and the advertised hop count are reinitialized when a route advertisement is received for a destination with a greater sequence number. AOMDV finds multiple paths which are node-disjoint or link disjoint routes. Each RREQs arriving via a different neighbour of the source defines a node-disjoint path. To find node-disjoint routes, each node does not immediately reject duplicate RREQs. This is because nodes cannot broadcast duplicate RREQs, so any two RREQs arriving at an intermediate node via a different neighbour of the source could not have traversed the same node. Destination only replies to RREQs arriving via unique neighbours in an order to get multiple linkdisjoint routes. After the first hop, the RREPs follow the reverse paths, which are node disjoint and thus linkdisjoint. Each RREP may intersect at an intermediate node, but each takes a different reverse path to the source to ensure link disjointness [12]. The advantage of using AOMDV is that it allows intermediate nodes to reply to RREQs, while still selecting disjoint paths. As AOMDV finds multiple paths and destination replies to multiple RREQs it has more routing overhead during route discovery.

\section{RELATED WORK}

\section{a. Approaches to find stable path.}

There are number of approaches to find a stable or reliable route for ad hoc networks. These approaches have different parameters like node residual energy, link expiration time, Stability of nodes, probabilistic link lifetime estimation and success rate of data transmission for node, link usage, and received signal strength [2]. 


\section{b. $\quad$ Choosing received signal strength as a parameter for stability}

Existing on demand routing protocols for ad hoc network does not take in its consideration the quality of link while deciding the route. If decision for selecting a route is based on received signal strength then the protocols can achieve the route which is more stable. Simulation in [6] shows good resemblance with actual implementation. Higher packet delivery ratio and lower routing load can be achieved if received signal strength is used as a parameter for finding the route [4][15].

\section{c. Cross-Layer Design}

In a cross layer design the parameter measured at physical layer can be made available to layers above it. The value measured at physical layer is passed to MAC layer and network layer. The received signal strength information sent by the physical layer may be stored either in routing table or neighbor table and can be used to make routing decisions. Such a cross layer design has been utilized to improve the performance of mobile ad hoc network [14].

Received signal strength of RREQ packet can be measured at physical level and based on that routing decisions can be made at routing layer [4]. If RREQ packet has a poor received signal strength then receiving node should not forward the RREQ to its neighbours, so weak link does not participate in the route. The received signal strength can be compared with a predetermined threshold to determine whether it is proper or not. The threshold can be a fixed threshold or an adaptive threshold whose value changes with changing speed of the nodes. The adaptive threshold also adapts to moving direction of the nodes.

The path loss of the link can be found by measuring the received signal strength by cross-layer approach [3]. The path loss measured for each link of an entire route can be added to find cumulative path loss of entire route. The average path loss for route can be found by dividing total path loss with hop count. Only the route which is having less path loss can be selected, so resulting route can be more stable.

The AODV-RSS in [5] determines paths that are long lived means, a route that can sustain for a longer time. The long-lived path routing algorithm uses the Received Signal Strength RSS and Received Signal Strength changing rate $\Delta$ RSS to predict the link available time (LAT) between two mobile nodes. AODV-RSS can improve the route quality in route connection time, and route reestablishment frequency.

The Route Stability based QOS Routing (RSQR) protocol proposed in [7] which is an extension of QOS routing with throughput and delay constraints. In order to guarantee the suitable data path for adequate longer duration in MANET, an easy model has been proposed for measuring the link stability and route stability depending on received signal strength. Some additional fields in route request/ reply packets is taken into consideration so that the route stability information can be used to choose a route with increased Stability when compared to all possible routes among existing source destination pair.

\section{StABiLity ENHANCED AODV PRotocol}

AODV broadcast RREQ (route request) packet to all neighbours in order to determine the route. The destination address is checked by neighbours and accordingly they forward RREQ to their neighbours. A RREQ packet traversing from different path arrives at the destination. The path with minimum hop is selected as a route [11]. The route found by AODV may have weak links which may lead to frequent route failures [6]. Frequent route failures will increase the number of route discoveries and in turn increase the routing overhead of the network.

The stability enhanced AODV routing protocol [4] [15] focuses on minimizing the routing overhead of the network. Received signal strength of incoming RREQ packet is measured and utilized to determine the route. The received signal strength information can be passed to routing layer using cross layer design. A RREQ packet is only forwarded if the link has sufficient received signal strength. So, the links with lower received signal strength i.e. weak links may not participate in formation of route.

A predetermined threshold is used to decide whether the link has the sufficient received signal strength or not. The threshold to be used can be a fixed value which can be determined based on performance.

Stability enhanced AODV protocol compares received signal strength of link with predetermined threshold which is the desired value of the received signal strength. The RREQ is only forwarded if the received signal strength of the link is greater than the threshold, so links having lower received signal strength may not participate in formation of route. The route found by fixed threshold method is stable and remains active for longer time. Reduced route discovery and reduced link failures can be achieved by fixed threshold, but overall 
performance may not be satisfactory due to increased no of hops in stable route. The increased no of hops increases the end to end delay and reduces the throughput of the network.

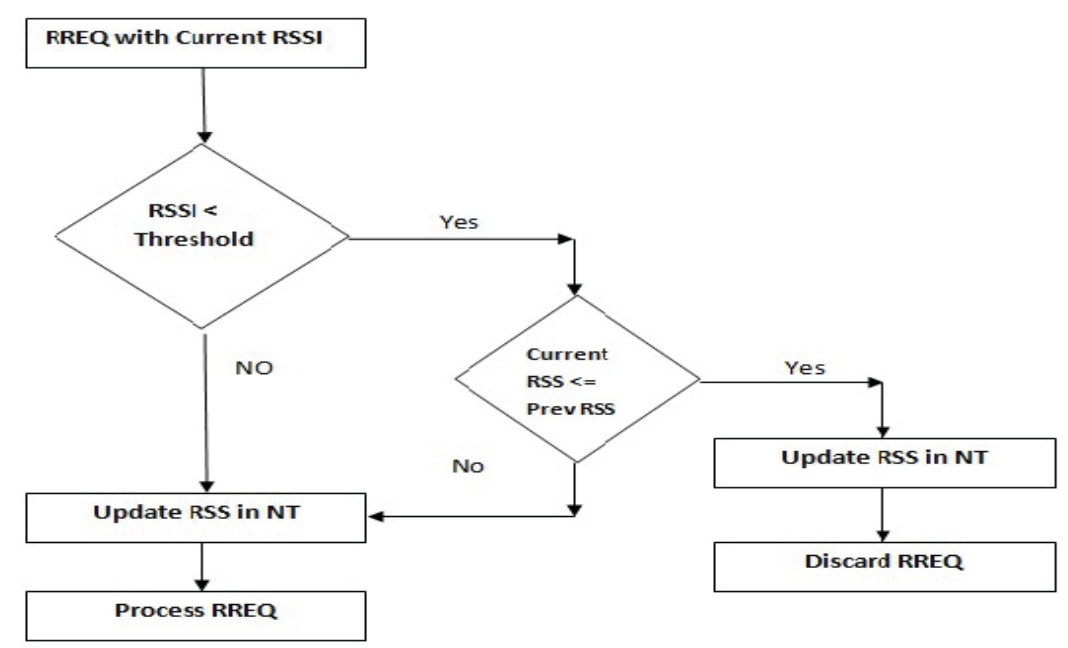

Fig. 1. Processing RREQ in Stability enhanced AODV

To improve the performance the adaptive threshold is used. Which uses different values of threshold with reference to moving speed of the nodes, in addition it also considers moving direction of nodes. Figure 1 shows the received signal strength of link is stored in the Neighbour table (NT) against the address of the neighbouring node from which it is received. So, whenever node receives RREQ from neighbours the current received signal strength is checked against the previous received signal strength stored in the table. If the current received signal strength is greater than the previous received signal strength, it indicates that the nodes are approaching towards each other. If the current received signal strength is lower than previous received signal strength, it indicates that the nodes are moving away from each other.

Whenever nodes receive RREQ from their neighbours its received signal strength is checked against a threshold. If the received signal strength is lower than the threshold but the nodes are approaching then nodes will forward the RREQ. If the received signal strength is lower than the threshold and the nodes are moving away from each other then the nodes discards the RREQ. If the received signal strength is greater than the threshold then the nodes forwards RREQ.

\section{STABILITy ENHANCED AOMDV PROTOCOL}

AOMDV is an on-demand routing protocol which broadcast RREQ (route request) packet to all neighbours in order to determine the route. Intermediate nodes checks destination address and accordingly they forward RREQ to their neighbours. A RREQ packet traversing from different path arrives at the destination. RREP (route reply) packet is send from receiver upon receiving RREQ [12]. AOMDV finds multiple paths for a single source destination pair. Those routes may have weak links which may lead to frequent route failures [6]. Frequent route failures will increase the number of route discoveries and in turn increase the routing overhead of the network.

Minimization of routing overhead of the network is primary focus of the stability enhanced AOMDV protocol. The received signal strength of the link is determined by measuring received signal strength of RREQ packet. Received signal strength information measured at physical layer is used at routing layer through cross layer design. A RREQ packet is only forwarded if the link has sufficient received signal strength. So, the links with lower received signal strength i.e. weak links may not participate in formation of route.

A predetermined threshold is used to decide whether the link has the sufficient received signal strength or not. The threshold to be used can be a fixed value which can be determined based on performance. 


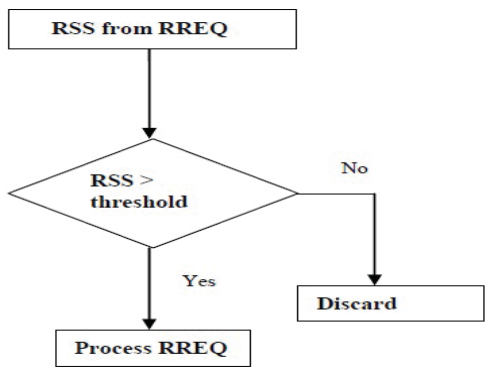

Fig. 2. Processing RREQ in Stability enhanced AOMDV

As shown in Figure 2 stability enhanced AOMDV protocol compares received signal strength of RREQ with predetermined threshold. The threshold value is nothing but the desired value of the received signal strength for link. If the received signal strength from the RREQ is greater than the threshold then RREQ packet will be processed else it will be discarded. In this way it takes care that no weak links can participate in formation of route. The route found by this method is stable and remains active for longer time.

With added stability to the route number of route failures can be reduced which can help in reducing routing load of the network. The method which is applied here finds the route which can contains increased number hops compared to route in existing AOMDV. The increase in number of hops can also increase end to end delay of the network. An additional delay can also affect throughput of the network.

\section{Simulation EnVIRONMENT AND ReSUlt ANALYSIS}

Network simulator 2 is a simulation tool which has been used to simulate both "stability enhanced AODV" and "stability enhanced AOMDV" protocols. NS-2.34 version is used which has support for simulating a multi-hop wireless ad-hoc network .49 nodes are deployed in an area of $1000 \times 1000 \mathrm{~m}^{2}$. Each point in result is an average of 10 seeds. The other simulation parameters are summarized in table I.

Table 1. Simulation parameters

\begin{tabular}{|l|l|}
\hline \multicolumn{1}{|c|}{ Parameters } & \multicolumn{1}{c|}{ Values } \\
\hline Transmission range & 250 meter \\
\hline Mobility model & Random Way point \\
\hline Propagation model & Two Ray Ground \\
\hline MAC Layer & 802.11 \\
\hline Simulation time & $200 \mathrm{sec}$ \\
\hline Transmission protocol & TCP \\
\hline Routing protocol & AODV Fixed, AODV Adaptive, Stability Enhanced AOMDV \\
\hline Packet Size & 512 bytes \\
\hline
\end{tabular}

We tested following three cases

1. Stability Enhanced AODV with fixed threshold (AODV Fixed)

2. Stability Enhanced AODV with adaptive threshold (AODV Adaptive)

3. Stability Enhanced AOMDV.

The fixed threshold value of $-60 \mathrm{db}$ is used for "Stability Enhanced AODV" Fixed threshold version and "Stability Enhanced AOMDV". They are tested for different speed in the range of $5 \mathrm{~m} / \mathrm{s}$ to $25 \mathrm{~m} / \mathrm{s}$. For "Stability Enhanced AODV" Adaptive version different threshold values (-63.66 db, -61.64 db, -60 db ,-59.37 $\mathrm{db},-56.89 \mathrm{db})$ are used for the speeds $(5 \mathrm{~m} / \mathrm{s}, 10 \mathrm{~m} / \mathrm{s}, 15 \mathrm{~m} / \mathrm{s}, 20 \mathrm{~m} / \mathrm{s}, 25 \mathrm{~m} / \mathrm{s})$ respectively. It also takes moving direction of the nodes into consideration.

\section{a. Packet Delivery Ratio}

The packet delivery ratio is parameter which describes efficient delivery of data packets. It is number of packets received per number of packet sent. Fig. 3 shows the packet delivery ratio for stability enhanced AOMDV and both the versions of stability enhanced AODV. The stability enhanced AOMDV seem to achieve better 
performance when nodes are moving at higher speed. Back up route in stability enhanced AOMDV helps it achieve a better packet delivery ratio.

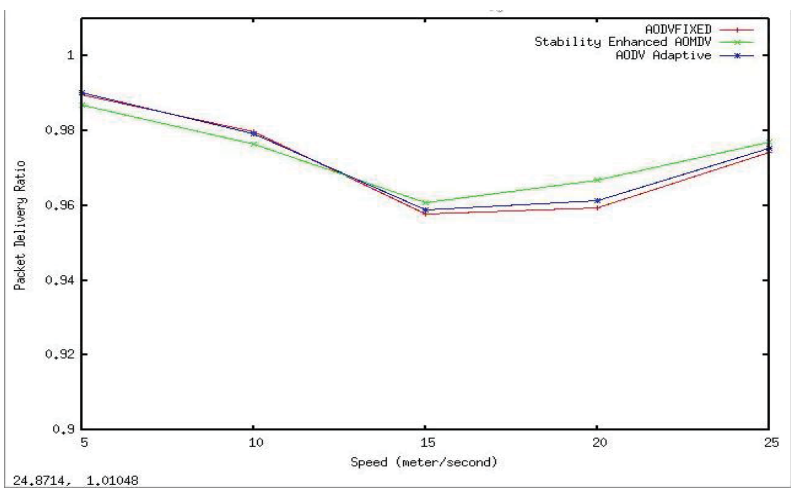

Fig. 3. Shows packet delivery ratio against speed of nodes

\section{b. Normalized Routing Load}

The normalized routing load or routing overhead of the network can be measured as number of routing packets sent per number of data packet received. From Fig 4 it can be observed that stability enhanced AOMDV has more routing overhead compared to both the versions of stability enhanced AODV. Stability enhanced AOMDV flood the network with RREQ packets in order to find multiple routes, which increases its routing load.

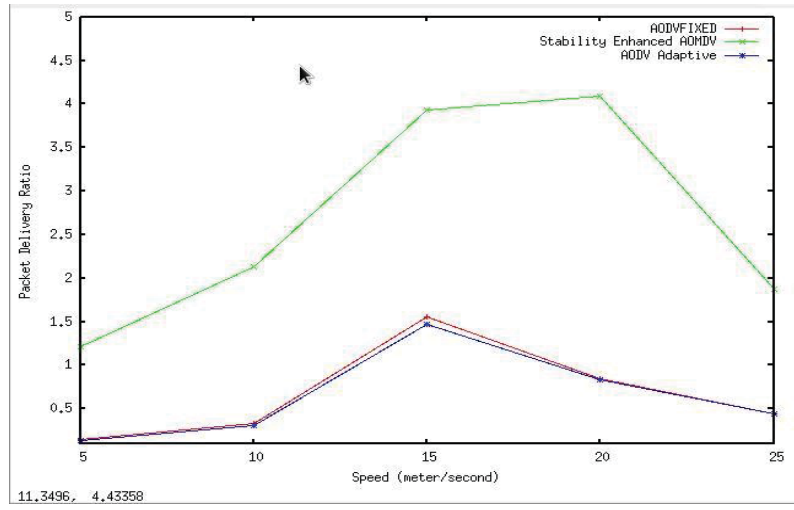

Fig. 4. Shows normalized routing load against speed of nodes

\section{c. Average end to End Delay}

The average end to end delay is the average of delay occurred in transmitting each data packet. From Fig 5 we can observe that stability enhanced AOMDV has lower average end to end delay compared to both the versions of stability enhanced AODV. The provision of alternate route in stability enhanced AOMDV is useful in case of route failure. Lower route discovery latency in stability enhanced AOMDV reduces the delay. 


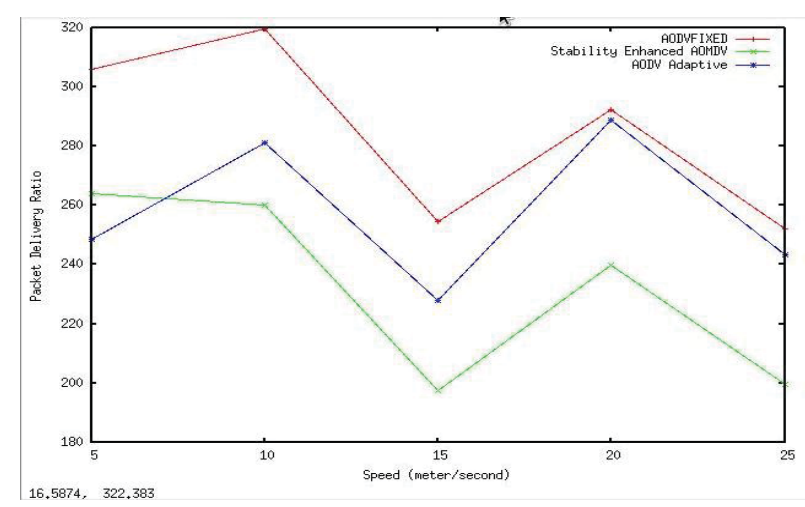

Fig. 5. Shows end to end delay against speed of nodes

\section{d. Throughput}

The throughput is total data transmitted per second. It can be observed form Fig 6 that both the versions of stability enhanced AODV achieve higher throughput than stability enhanced AOMDV. Stability enhanced AOMDV has lower end to end delay but it has higher routing load compared to Stability enhanced AODV which decreases the throughput. We can also observe that Adaptive version of Stability enhanced AODV achieves better throughput compared to fixed version as it considers moving direction of the nodes.

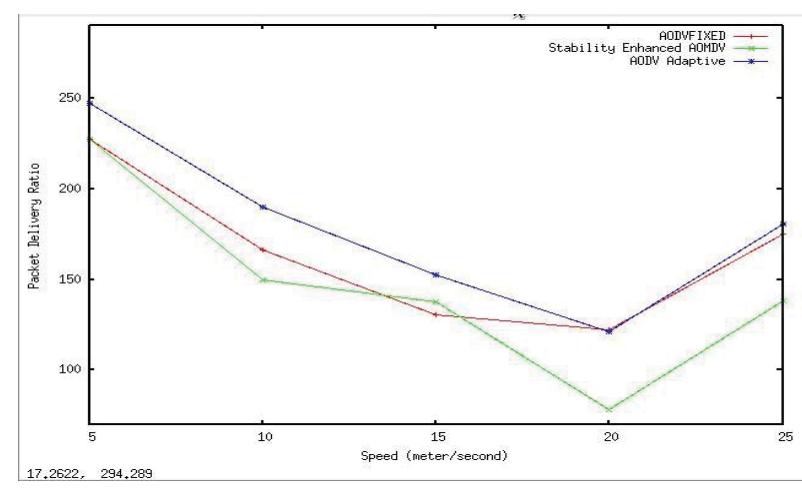

Fig. 6. Shows throughput against speed of nodes

\section{CONCLUSION}

In this paper we have done performance comparison between two versions of stability enhanced AODV and stability enhanced AOMDV protocol. Results suggest that both the versions of stability enhanced AODV has lower routing load compared to stability enhanced AOMDV protocol. Stability enhanced AOMDV has to find multiple paths in single route discovery, so it floods the network with RREQ packets. The route discovery procedure for stability enhanced AOMDV contains more routing packets compared to stability enhanced AODV. Due to lower routing load both the versions of stability enhanced AODV achieves better packet delivery ratio and throughput than stability enhanced AOMDV. We can also observe that stability enhanced AOMDV has lower End to End delay compared to stability enhanced AODV protocol. Stability enhanced AOMDV has provision of backup rote which avoids route discovery when primary route fails. It saves the delay which occurs in finding a new route. We can achieve better routing load, higher packet delivery ratio and higher throughput in Stability enhanced AODV compared to Stability enhanced AOMDV, but Stability enhanced AOMDV has lower end to end delay.

\section{REFERENCES}

[1] H.D.Trung, W.Benjapolakul, P.M.Duc, "Performance evaluation and comparison of different ad hoc routing protocols", Department of Electrical Engineering, Chulalongkorn University, and Bangkok, Thailand May 2007.

[2] Hadi Sargolzaey, Ayyoub Akbari Moghanjoughi and Sabira Khatun," A Review and Comparison of Reliable Unicast Routing Protocols For Mobile Ad Hoc Networks", International Journal of Computer Science and Network Security (IJCSNS), VOL.9 No.1 January 2009. 
[3] Boumedjout Amel, Mekkakia Maaza Zoulikha, "Routing Technique with Cross-layer Approach in Ad Hoc Network", IEEE, 2009

[4] B.Ramchandran and S. shanmugavel, "Received Signal Strength-based Cross-layer design for Mobile Ad Hoc Networks", IETE Technical Review, Volume 25, Issue 4, JUL-AUG 2008.

[5] Ruay-Shiung Chang and Shing-Jiuan Leu, "Long-lived Path Routing With Received Signal Strength for Ad Hoc Networks", IEEE International Symposium on Wireless Pervasive Computing, January 2006.

[6] Kwan-Wu Chin, John Judge, Aidan Williams and Roger Kermode, "Implementation Experience with MANET Routing Protocols", ACM SIGCOMM Computer Communications Review, Volume 32, Number 5, November 2002.

[7] Nityananda Sarma and Sukumar Nandi, "Route Stability Based QoS Routing in Mobile Adhoc Networks", Wireless Personal Communication, Vol-54, pp-203-224, 2010.

[8] Fang Xie, Lei Du, Yong Bai, Lan Chen, "Energy Aware Reliable Routing Protocol for Mobile Ad-Hoc Networks", IEEE Communication Society, WCNC 2007proceedings.

[9] Zhao Cheng, Wendi B. Heinzelman, "Discovering long lifetime routes in mobile ad hoc networks", Elsevier Ad Hoc Networks 6, 2008, p661-674.

[10] Xie Xiaochuan, Wei Gang, Wu Keping, Wang Gang, Jia Shilou, "Link reliability based hybrid routing for tactical mobile ad hoc network", Elsevier Journal of Systems Engineering and Electronics Vol. 19, No. 2, 2008, pp.259-267.

[11] Charles E. Perkins, Elizabeth M. Belding-Royer, Samir R. Das, February 2003, "Ad hoc On-Demand Distance Vector (AODV) Routing draft-ietf-manet-aodv-13.txt,”, Mobile Ad Hoc Networking Working Group, INTERNET DRAFT.

[12] M.K.Marina and S.R.Das, "On-Demand Multipath distance vector routing in ad hoc Networks" in: Proceedings of 9th IEEE International Conference on Network Protocols (ICNP), 2001.

[13] The ns Manual, Formerly ns Notes and Documentation.

[14] Chintan Bhavsar,"A Survey On Cross-Layer Reliable Routing Protocols in MANETs", International Journal of Innovative Research in Science, Engineering and Technology, Vol 03, Issue 4, April 2014.

[15] Chintan Bhavsar, Mayur M. Vegad, Sunil A. Bakhru, "Performance Comparison of Stability Enhanced AODV and AOMDV protocols for MANETs", IJETAE-International Journal of Emerging Technology and Advanced Engineering, Volume 2, Issue 5, May 2012. 